\title{
EXCHANGE BIAS IN NANO-FERRIHYDRITE
}

D.A. Balaev ${ }^{1,2}$, A.A. Krasikov, ${ }^{2, *}$, A.A. Dubrovskiy ${ }^{1,4}$, S.I.Popkov ${ }^{1,2}$, S.V. Stolyar ${ }^{1,2}$, R.S. Iskhakov ${ }^{1}$, V.P. Ladygina ${ }^{3}$, R.N. Yaroslavtsev ${ }^{2}$.

${ }^{*}$ Corresponding author e-mail: $\underline{\text { KAA3000@yandex.ru }}$

1 - Kirensky Institute of Physics, Russian Academy of Sciences, Siberian Branch, Krasnoyarsk, 660036, Russia.

2 - Siberian Federal University, Krasnoyarsk, 660041, Russia.

3 - Presidium of Krasnoyarsk Scientific Center, Russian Academy of Sciences, Siberian Branch, Krasnoyarsk, 660036, Russia.

${ }^{4}$ - International Laboratory of High Magnetic Fields and Low Temperatures, Gajowicka 95, 53421 Wroclaw; Poland.

We report the results of investigations of the effect of cooling in an external magnetic field starting from the temperature over superparamagnetic blocking temperature $T_{B}$ on the shift of magnetic hysteresis loops in systems of ferrihydrite nanoparticles from $\sim 2.5$ to $\sim 5 \mathrm{~nm}$ in size with different $T_{B}$ values. In virtue of high anisotropy fields of ferrihydrite nanoparticles and open hysteresis loops in the range of experimentally attainable magnetic fields, the shape of hysteresis loops of such objects in the field-cooling mode is influenced by the minor hysteresis loop effect. A technique is proposed for distinguishing the exchange bias effect among the effects related to the minor hysteresis loops caused by high anisotropy fields of ferrihydrite particles. The exchange bias in ferrihydrite is stably observed for particles no less than $3 \mathrm{~nm}$ in size or with $\mathrm{T}_{\mathrm{B}}$ over $40 \mathrm{~K}$, and its characteristic value increases with the particle size.

\section{Introduction}

The well-known phenomenon of exchange bias in the ferromagnet/antiferromagnet (FM/AF) structures, i.e., the shift of a magnetic hysteresis loop relative to the origin of coordinates, has still been intensively studied [1-4]. The effect consists in the following: the exchange coupling at the interface between AF and FM layers creates an additional magnetic anisotropy source, which manifests itself upon cooling the system in an external field starting from the temperature exceeding the Néel temperature of the antiferromagnet. The exchange bias has been observed in many types of materials containing the FM/AF interfaces, including magnetic nanoparticle systems [2-4]. The interesting and still understudied experimental fact is the presence of exchange bias in single-phase AF nanoparticles, e.g., $\mathrm{NiO}$ [5-7] and $\mathrm{CuO}$ [8-13]. In such objects, the FM «phase» can be an uncompensated magnetic moment of particles, which is caused by defects and incomplete compensation of the AF sublattices. On the other hand, the spin-glass-like behavior of particle surface atoms exchange-coupled with the AF- 
ordered «core» can also cause the observed exchange bias. Finally, according to the numerous numerical calculations of the micromagnetic structure, the multi-sublattice states can form in a small AF [14]; the magnetization switching in such a particle upon cooling in an external field also leads to asymmetry of the hysteresis loop.

Nanoparticles of ferrihydrite with the nominal formula $5 \mathrm{Fe}_{2} \mathrm{O}_{3} \cdot 9 \mathrm{H}_{2} \mathrm{O}$ and its biogenic analog horse spleen ferritin exhibit the AF ordering [15-18]. The shift of the hysteresis loop in these particles upon cooling in a magnetic field starting from the temperature over the superparamagnetic (SP) blocking temperature $T_{B}$ was reported by many authors $[15,19-26]$. At the same time, it was shown that the magnetic hysteresis loops of these materials at $T<T_{B}$ are open (minor) in external fields of 60-90 kOe used in the experiments [19, 20, 22-26]. The question about the existence of exchange bias in ferrihydrite remains unanswered [26], since the observed magnetic hysteresis shift can be an artifact. Silva et al. [26] attributed the discussed effect in ferritin and ferrihydrite, whose $T_{B}$ value is no more than $\sim 30 \mathrm{~K}$ [26-28], to the existence of high-energy barriers for the uncompensated magnetic moment, which are induced by the effective magnetic anisotropy of particles upon cooling in magnetic field; i.e., the loop shift is, in fact, analogous to the minor loop effect.

In this work, we analyze the experimental data on observation of the hysteresis loop shift in ferrihydrite samples, compare this effect to the behavior of minor hysteresis loops, and demonstrate the existence of exchange bias in the system. The investigations were carried out on ferrihydrite samples prepared by different techniques and characterized by different particle sizes and, consequently, different SP blocking temperatures.

\section{Experimental}

\subsection{Samples}

A technique for preparation nano-ferrihydrite formed during the vital activity of bacteria was described in detail in $[29,30,23]$. Hereinafter, the initial sample is denoted as b-fh. Lowtemperature $\left(140-200^{\circ} \mathrm{C}\right)$ annealing of ferrihydrite leads to an increase in the size of particles due to their partial agglomeration [24, 31, 32]. The investigated bacterial ferrihydrite samples annealed at different temperatures for different times are denoted as b-fh-a and b-fh-a*.

Synthetic ferrihydrite was prepared using the technique described in [33]. The roomtemperature continuously intermixed $\mathrm{NaOH}$ solution was added to the iron chloride $\left(\mathrm{FeCl}_{3}\right)$ solution to obtain a neutral $\mathrm{pH}$ value. The precipitate was collected on a filter, washed, and dried at room temperature. The synthetic ferrihydrite sample is denoted as s-fh.

Table 1 gives average particle size $<\mathrm{d}>$ for the investigated samples in accordance with the transmission electron microscopy (TEM) data obtained on a Hitachi HT7700 microscope of 
the Center of Collective Use, Krasnoyarsk Scientific Center, Russian Academy of Sciences, Siberian Branch. Typical microphotographs were presented in $[32,34])$. According to our data, the $\left\langle\mathrm{d}>\right.$ values are consistent with those obtained from the magnetization curves at $\mathrm{T}>\mathrm{T}_{\mathrm{B}}[23$, $24,32]$.

In addition, the samples were characterized using Mossbauer spectroscopy. The results obtained agree well with the parameters of ferrihydrite model spectra from [29, 31, 32]. Upon annealing, no features indicative of new phases of iron hydroxide or oxide were observed.

\subsection{Magnetic measurements}

The magnetic measurements were performed on a vibrating sample magnetometer with a superconducting solenoid [35]. The investigated powder sample was fixed in a measuring capsule using paraffin (the data obtained were corrected to the paraffin diamagnetic signal). Temperature dependences of the magnetic moment $\mathrm{M}(\mathrm{T})$ were measured under zero-field cooling (ZFC) and field cooling (FC) at $\mathrm{H}=1 \mathrm{kOe}$. The $\mathrm{ZFC}$ hysteresis $\mathrm{M}(\mathrm{H})$ dependences were measured at $\mathrm{T}=4.2 \mathrm{~K}$ up to different maximum magnetic fields $\mathrm{H}_{\max }$ with a gradual increase in the $\mathrm{H}_{\max }$ value to $75 \mathrm{kOe}$. The $\mathrm{FC} \mathrm{M}(\mathrm{H})$ dependences were measured upon sample cooling in external fields of $\mathrm{H}_{\mathrm{cool}}=15,30,45$, and $60 \mathrm{kOe}$ hereinafter denoted as FC $15 \mathrm{kOe}, \mathrm{FC} 30 \mathrm{kOe}$, etc. starting from a temperature of $120 \mathrm{~K}$, which a priori exceeds the observed $\mathrm{T}_{\mathrm{B}}$ values. After cooling in a field of up to $4.2 \mathrm{~K}$, the $\mathrm{M}(\mathrm{H})$ dependence was measured at the multiple external field cycling within $\pm \mathrm{H}_{\text {cool }}$; in some agreed cases, after cooling in fields of $\mathrm{H}_{\text {cool }}=30$ and $45 \mathrm{kOe}$, the external field was changed within $\pm 60 \mathrm{kOe}$. In addition, minor hysteresis loops were measured in accordance with the following scheme: in the ZFC mode, an external field of $\mathrm{H}_{\max }=+75 \mathrm{kOe}$ was applied and then the $\mathrm{M}(\mathrm{H})$ dependences were measured at the multiple external field cycling within $\pm 15 \mathrm{kOe}$ (this regime is denoted as ML $75 \mathrm{kOe}$ ). The external field variation rate was $50 \mathrm{Oe} / \mathrm{s}$ and, according to our data, the twofold increase in this parameter almost did not affect the $\mathrm{M}(\mathrm{H})$ dependences and investigated parameters, including coercivity $\mathrm{H}_{\mathrm{C}}$ and residual magnetic moment $\mathrm{M}_{\mathrm{R}}$.

\section{Results and discussion}

The ZFC and FC M(T) dependences are presented in Fig. 1. The maximum in the ZFC $\mathrm{M}(\mathrm{T})$ dependences and the bifurcation of the $\mathrm{M}(\mathrm{T})$ dependences for different magnetic prehistories are typical of nanoparticle systems with the SP behavior. Table 1 gives the SP blocking temperatures $\mathrm{T}_{\mathrm{B}}$ corresponding to the maximum of the $\mathrm{ZFC} \mathrm{M}(\mathrm{T})$ dependence. The average blocking temperatures $\left\langle\mathrm{T}_{\mathrm{B}}\right\rangle$ given in Table 1 were determined from the data illustrated 
in Fig. 1 and correspond to the maximum of the function $\mathrm{d}\left(\mathrm{M}(\mathrm{T})_{\mathrm{ZFC}}-\mathrm{M}(\mathrm{T})_{\mathrm{FC}}\right) / \mathrm{dT}$ [36]. It can be seen that an increase in the average particle size is consistent with an increase in $\left\langle\mathrm{T}_{\mathrm{B}}\right\rangle$ and $\mathrm{T}_{\mathrm{B}}$.

Along with the family of ZFC hysteresis loops measured in fields up to different $\mathrm{H}_{\max }$ values, Fig. 2a shows the $\mathrm{FC} \mathrm{M}(\mathrm{H})$ dependences obtained at $\mathrm{H}_{\text {cool }}=15,30$, and $45 \mathrm{kOe}$ for sample b-fh-a. For the FC mode, we introduce the coercivities $\mathrm{H}_{\mathrm{CL}}$ and $\mathrm{H}_{\mathrm{CR}}$ according to the criterion $\mathrm{M}=0$ (see inset in Fig. 2a). It can be seen that the FC hysteresis loops are shifted along the $\mathrm{H}$ and $\mathrm{M}$ axes and the $\mathrm{H}_{\mathrm{CL}}$ values are approximately equal for different $\mathrm{H}_{\mathrm{cool}}$, which is indicative of the possible existence of exchange bias.

However, as follows from the data shown in Fig. 2a, the $\mathrm{M}\left(\mathrm{H}=\mathrm{H}_{\text {cool }}\right)$ values do not coincide after external field cycling by the scheme $\mathrm{H}_{\text {cool }} \rightarrow-\mathrm{H}_{\text {cool }} \rightarrow \mathrm{H}_{\text {cool }}$. In addition, note the trend to shifting the FC “coercivity" $\mathrm{H}_{\mathrm{CR}}$ to the left with decreasing $\mathrm{H}_{\mathrm{cool}}$ (inset in Fig. 2). This is typical of all the investigated samples (see the details of the $\mathrm{M}(\mathrm{H})$ dependences near the origin of coordinates in Figs. $2 b$ and $2 c$ ). The exchange bias $\mathrm{H}_{\mathrm{EB}}$ is usually determined as $\mathrm{H}_{\mathrm{EB}}=-$ $\left(\mathrm{H}_{\mathrm{CL}}+\mathrm{H}_{\mathrm{CR}}\right) / 2$ [2-4]. The $\mathrm{H}_{\mathrm{EB}}$ value determined in this way for the data from Fig. 2 decreases with increasing $\mathrm{H}_{\text {cool. }}$. This can be explained by the fact that the important role in the behavior of the $\mathrm{FC} \mathrm{M}(\mathrm{H})$ dependences is played by the minor loop effect [4].

The set of data obtained showed that this effect is the most pronounced at the multiple field cycling within $\pm \mathrm{H}_{\text {cool }}$ already after cooling in the field $+\mathrm{H}_{\text {cool }}$. The example of evolution of the $\mathrm{M}(\mathrm{H})$ dependences for sample b-fh-a at these regimes is shown in Figs. 3a, 3c, and 3d. The minor hysteresis loop effect in its pure form corresponds to regime ML $75 \mathrm{kOe}$ (see Section 2.2). Figure $3 b$ shows the $\mathrm{M}(\mathrm{H})$ dependences obtained in this regime, i.e., at the variation in the external field within \pm 15 kOe. The qualitative comparison of the data from Fig. 3b and Figs. 3a, $3 \mathrm{c}$, and $3 \mathrm{~d}$ shows that the evolution of the $\mathrm{M}(\mathrm{H})$ dependences at the sequential field cycling is approximately the same for different regimes.

Figure $3 e$ summarizes the data on the evolution of parameters $H_{C L}$ and $H_{C R}$ upon variation in the number of field cycles $\mathrm{n}$. The larger change (a decrease in the absolute value) is observed in the parameter $\mathrm{H}_{\mathrm{CL}}$ both after field cooling and in regime ML $75 \mathrm{kOe}$. The dependence of the parameters $\mathrm{H}_{\mathrm{CL}}$ and $\mathrm{H}_{\mathrm{CR}}$ on $\mathrm{n}$ can reflect the behavior of the minor hysteresis loops or be an intrinsic property of AF/FM structures, which is called the training effect [4]. In the latter case, the training effect is determined by the properties of the AF material and is caused by spin structure rearrangement $[4,37]$. This effect is characterized by the empirical dependence $\mathrm{H}_{\mathrm{EB}} \sim \mathrm{n}^{-1 / 2}$ valid at $\mathrm{n} \geq 2$, which was multiply confirmed in experiments with different systems (see, for example, [4, 37, 38]). The data shown in Fig. 3e and obtained by us on other samples are not described by this empirical dependence. This evidences for the dominant contribution of the minor hysteresis loop effect to the evolution of $\mathrm{M}(\mathrm{H})$ dependences at the field cycling. The 
behavior of the residual magnetic moment $M_{R}$ (the upper and lower $M$ values at $H=0$ ) for the FC hysteresis loops at the field cycling is analogous to the dependences of $\mathrm{H}_{\mathrm{CL}}$ and $\mathrm{H}_{\mathrm{CR}}$ on $n$ (Fig. 3e).

On the other hand, the $\mathrm{H}_{C L}$ values at $n=1$ noticeably exceed the $\mathrm{H}_{\mathrm{C}}\left(\mathrm{H}_{\max }=75 \mathrm{kOe}\right)$ value and weakly depend on $\mathrm{H}_{\text {cool }}$ (Fig. 2). This indirectly indicates the effect of exchange bias on the observed shift of the hysteresis loop, taking into account that the minor hysteresis loop effect manifests itself already during field cycling. To separate the exchange bias effect from the contribution of minor hysteresis loops, it is reasonable to compare the $\mathrm{H}_{\mathrm{CL}}$ values and coercivities $\mathrm{H}_{\mathrm{C}}$ observed under the $\mathrm{ZFC}$ conditions. Since the magnetic hysteresis loops are open, which can be seen in Fig. 2a for the ZFC mode, it is necessary to extrapolate the $\mathrm{H}_{\mathrm{C}}\left(\mathrm{H}_{\max }\right)$ dependence to the high-field region in order to determine the coercivity of the limit hysteresis loop $\mathrm{H}_{\text {Cinf }}$ (in fact, the $\mathrm{H}_{\mathrm{C}}$ value at "infinite" $\mathrm{H}_{\max }$ ).

In [26], based on the concepts of field dependence of the energy barriers caused by the magnetic anisotropy overcome by the magnetic moment of a particle, the following expression for the $\mathrm{H}_{\mathrm{C}}\left(\mathrm{H}_{\max }\right)$ dependence was proposed:

$$
\mathrm{H}_{\mathrm{C}}\left(\mathrm{H}_{\max }\right)=\mathrm{H}_{\mathrm{Cinf}}\left[1-\left(\mathrm{H}^{*} / \mathrm{H}_{\max }\right)^{\beta}\right] .
$$

This expression is valid at the fields noticeably higher than $\mathrm{H}^{*}$ and the exponent $\beta$ is determined by the structure of energy barriers [26]; in the case of ferrihydrite, the authors obtained the value of $\beta \approx 1.5$. The set of results obtained in this study agrees well with the experimental data on $\mathrm{H}_{\mathrm{C}}\left(\mathrm{H}_{\max }\right)$ at the fields over $\sim 2 \cdot \mathrm{H}^{*}$ at $\beta \approx 1.5$. Fitting of the $\mathrm{H}_{\mathrm{C}}\left(\mathrm{H}_{\max }\right)$ dependences yielded the $\mathrm{H}_{\text {Cinf }}$ values for the investigated samples (see Table 1 and figure 4). The dependences of the residual magnetic moment $\mathrm{M}_{\mathrm{R}}$ on $\mathrm{H}_{\max }$ under the $\mathrm{ZFC}$ conditions behave similarly to dependence (1):

$$
\mathrm{M}_{\mathrm{R}}\left(\mathrm{H}_{\max }\right)=\mathrm{M}_{\text {Rinf }}\left[1-\left(\mathrm{H}^{*} / \mathrm{H}_{\max }\right)^{\beta}\right],
$$

where $\mathrm{M}_{\mathrm{Rinf}}$ is the residual magnetic moment at $\mathrm{H}_{\max }=\infty$; the obtained $\mathrm{M}_{\text {Rinf }}$ values are given in Table 1.

Figure 4 shows the $\mathrm{H}_{\mathrm{C}}\left(\left|\mathrm{H}_{\max }\right|\right)$ dependences (on the top) and $\mathrm{M}_{\mathrm{Rinf}}\left(\left|\mathrm{H}_{\max }\right|\right)$ dependences (in the bottom) for samples s-fh, b-fh, and b-fh-a. The results of fitting by dependences (1) and (2) are shown by solid lines and the extrapolated $\mathrm{H}_{\text {Cinf }}$ and $\mathrm{M}_{\text {Rinf }}$ values, by horizontal dashed lines; the line thickness approximately corresponds to the error of determination of these values. In addition, the data on $\mathrm{H}_{\mathrm{CL}}, \mathrm{H}_{\mathrm{CR}}$, and $\mathrm{M}_{\mathrm{R}}$ (the upper and lower values) obtained under the $\mathrm{FC}$ conditions at different $\mathrm{H}_{\text {cool }}$ are presented; the values on the abscissa axis correspond to the $\mathrm{H}_{\mathrm{cool}}$ 
values. Each row of experimental points for the FC mode with the same abscissa reflects the evolution of these parameters at the field cycling within $\pm \mathrm{H}_{\text {cool }}$ (see also Figs. 2 and 3).

It can be seen in Fig. 4 that in samples s-fh and b-fh-a the detected $H_{C L}$ and $M_{R}$ values noticeably exceed the extrapolated $\mathrm{H}_{\text {Cinf }}$ and $\mathrm{M}_{\text {Rinf }}$ values. In view of the aforesaid, we can state that the hysteresis loop shift in these samples is caused not only by the minor hysteresis loop effect.

The data for the field cycling within $\pm \mathrm{H}_{\mathrm{cool}}$ after cooling can be compared with the $\mathrm{H}_{\mathrm{C}}\left(\mathrm{H}= \pm \mathrm{H}_{\text {cool }}\right)$ values. The $\mathrm{H}_{\mathrm{CR}}$ values for the $\mathrm{FC}$ mode are smaller than the corresponding $\mathrm{H}_{\mathrm{C}}\left(\mathrm{H}=+\mathrm{H}_{\text {cool }}\right)$ values for the $\mathrm{ZFC}$ mode and the $\left|\mathrm{H}_{\mathrm{CL}}\right|$ values become smaller than $\left|\mathrm{H}_{\text {Cinf }}\right|$ already at $\mathrm{n}=2$, although they do not attain the coercivity $\left|\mathrm{H}_{\mathrm{C}}\left(\mathrm{H}=-\mathrm{H}_{\mathrm{cool}}\right)\right|$ under the ZFC conditions at the multiple cycling. If after field cooling at $\mathrm{H}_{\text {cool }}=30$ and $45 \mathrm{kOe}$ the external field is changed for the larger value $( \pm 60 \mathrm{kOe})$, then the $\left|\mathrm{H}_{\mathrm{CL}}\right|$ and $\mathrm{H}_{\mathrm{CR}}$ values will also be somewhat higher than those for the field cycling in the ranges \pm 30 and $45 \mathrm{kOe}$ (Fig. 4a). These trends in the $\mathrm{H}_{\mathrm{CL}}$ and $\mathrm{H}_{\mathrm{CR}}$ behavior with respect to $\mathrm{H}_{\mathrm{C}}\left(\mathrm{H}= \pm \mathrm{H}_{\text {cool }}\right)$ are typical also of the data on the residual magnetic moment (Figs. 4b, 4d, and 4f) and characterize the minor hysteresis loop effect, since the $\mathrm{H}_{\text {cool }}$ and $\mathrm{H}_{\max }$ values are fairly far from the anisotropy field (hundreds of kOe) required for obtaining the closed hysteresis loop.

The largest difference between the $\mathrm{H}_{\mathrm{CL}}$ and $\mathrm{H}_{\text {Cinf }}$ values and, correspondingly, between

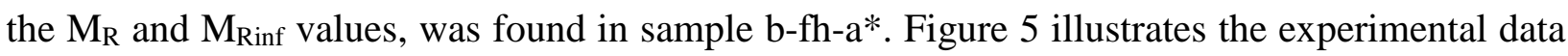
for this sample, including the $\mathrm{ZFC} \mathrm{M}(\mathrm{H})$ dependences and shifted hysteresis loop obtained at $\mathrm{H}_{\text {cool }}=30 \mathrm{kOe}$. The example of extrapolation of the $\mathrm{H}_{\mathrm{C}}\left(\left|\mathrm{H}_{\max }\right|\right)$ dependence together with the $\mathrm{H}_{\mathrm{CL}}$ values at $\mathrm{n}=1-5$ is presented in the lower inset in Fig. 5. In addition, the $\pm \mathrm{M}_{\text {Rinf }}$ and $\pm \mathrm{H}_{\text {Cinf }}$ values are plotted.

Thus, we can speak about the presence of exchange bias, at least, in samples s-fh, b-fh-a, and $b-f h-a^{*}$. Now, the question arises how to determine the exchange field $\mathrm{H}_{\mathrm{EB}}$. In our opinion, the well-known expression $\mathrm{H}_{\mathrm{EB}}=-\left(\mathrm{H}_{\mathrm{CL}}+\mathrm{H}_{\mathrm{CR}}\right) / 2$ cannot be used here, since the $\mathrm{H}_{\mathrm{CR}}$ values depend on the $\mathrm{H}_{\text {cool }}$ value due to the minor hysteresis loop effect. At the same time, as can be seen in Fig. 4 , the $\mathrm{H}_{\mathrm{CR}}$ values are close to the coercivity $\mathrm{H}_{\mathrm{C}}\left(\mathrm{H}_{\max }=+\mathrm{H}_{\text {cool }}\right)$ of the $\mathrm{ZFC}$ hysteresis loops. Therefore, it would be reasonable to believe that at the very high fields $\mathrm{H}_{\text {cool }}$, the parameter $\left(\left|\mathrm{H}_{\mathrm{CL}}\right|+\left|\mathrm{H}_{\mathrm{CR}}\right|\right)$ will be close to $2 \mathrm{H}_{\mathrm{Cinf}}$. This assumption is confirmed by the closeness of the $\left(\left|\mathrm{H}_{\mathrm{CL}}\right|+\left|\mathrm{H}_{\mathrm{CR}}\right|\right)$ values at $\mathrm{H}_{\mathrm{cool}}=45 \mathrm{kOe}, 60 \mathrm{kOe}($ at $\mathrm{n}=1)$, and $2 \mathrm{H}_{\mathrm{Cinf}}$, which follows from the data illustrated in Figs.4a, 4c, and 4e, as well as from the analogous values related to the residual magnetic moment (Figs. 4b, 4d, and 4f). In view of the aforesaid, we consider the exchange bias to be expressed as 


$$
\mathrm{H}_{\mathrm{EB}}=\left|\mathrm{H}_{\mathrm{CL}}\right|-\left|\mathrm{H}_{\mathrm{Cinf}}\right|
$$

The $\mathrm{H}_{\mathrm{EB}}$ values determined using Eq. (3) are given in Table 1. One can see the trend to an increase in the exchange bias with the particle size (the $\mathrm{H}_{\mathrm{EB}}$ value for sample b-fh is no more than the error of determination of $\mathrm{H}_{\text {Cinf }}$ ) or with the blocking temperature, which reflects an increase in the particle size more exactly.

In $[7,8]$, the dependences of $\mathrm{H}_{\mathrm{EB}}$ on size $\mathrm{d}$ of $\mathrm{NiO}$ and $\mathrm{CuO}$ particles in the ranges of 5-55 and 6.6-35 nm, respectively, were experimentally obtained. These dependences are characterized by an increase in $\mathrm{H}_{\mathrm{EB}}$ to certain size $\mathrm{d}(\sim 26 \mathrm{~nm}$ for $\mathrm{NiO}$ [7] and $\sim 12 \mathrm{~nm}$ for $\mathrm{CuO}$ [8]) with a subsequent decrease in $\mathrm{H}_{\mathrm{EB}}$ at larger sizes. In the framework of the classical approach, the exchange bias for the FM/AF structures is determined by the ratio between the parameters of an antiferromagnet (exchange constant $\mathrm{A}_{\mathrm{AF}}$ and anisotropy constant $\mathrm{K}_{\mathrm{AF}}$ ) and a ferromagnet (saturation magnetization $\mathrm{M}_{\mathrm{FM}}$ and FM layer thickness $\mathrm{d}_{\mathrm{FM}}[2,3]$ ):

$$
\mathrm{H}_{\mathrm{EB}}=2\left(\mathrm{~A}_{\mathrm{AF}} \mathrm{K}_{\mathrm{AF}}\right)^{1 / 2} / \mathrm{M}_{\mathrm{FM}} \mathrm{d}_{\mathrm{FM}} \text {. }
$$

The initial rise of the $\mathrm{H}_{\mathrm{EB}}(\mathrm{d})$ dependence was attributed by the authors of $[7,8]$ to a decrease in the $\mathrm{M}_{\mathrm{FM}}$ value with increasing particle size, which was determined by subtraction of the linear function $\mathrm{M}=\chi_{\mathrm{AF}} \mathrm{H}$, where $\chi_{\mathrm{AF}}$ is the magnetic susceptibility of an antiferromagnet, from the experimental $\mathrm{M}(\mathrm{H})$ dependence. In the case of ferrihydrite, the procedure is complicated by the difficulty of determination of the field in which the $\mathrm{M}_{\mathrm{FM}}$ approaches the saturation and the experimental $\mathrm{M}(\mathrm{H})$ dependence becomes a linear function of the field [39, 40]. However, a decrease in $\mathrm{M}_{\mathrm{FM}}$ with increasing AF nanoparticle size follows directly from the well-known Néel hypothesis about the relation $\mu_{\mathrm{unc}} \sim \mathrm{V}^{a}$ between uncompensated magnetic moment $\mu_{\mathrm{unc}}$ and particle volume $\mathrm{V}$, where the exponent $a$ is determined by the type of defects [41]. Numerous investigations of ferritin and ferrihydrite [15-17, 19, 22-24, 32, 42, 43] confirmed that the $a$ value is close to $1 / 2$. Under the assumption $\mathrm{M}_{\mathrm{FM}}=\mu_{\mathrm{und}} / \mathrm{V}$, we have $\mathrm{M}_{\mathrm{FM}} \sim \mathrm{V}^{-1 / 2}$. Then, taking into account expression (4), the growth of $\mathrm{H}_{\mathrm{EB}}$ with increasing ferrihydrite particle size can be understood at the qualitative level: $\mathrm{H}_{\mathrm{EB}} \sim \mathrm{V}^{1 / 2}$. The spin-glass-like state of surface atoms and their exchange coupling with the AF particle core can significantly change the obtained $\mathrm{H}_{\mathrm{EB}}(\mathrm{V})$ dependence.

On the other hand, at an AF particle size of a few nanometers, the parameters in the numerator of Eq. (4) can change. Indeed, a large number of the exchange bonds broken by surface defects can lead to the occurrence of the multi-sublattice states in an AF nanoparticle [14], which can also lead to the observed shift of the hysteresis loop. It would be reasonable to analyze the $\mathrm{H}_{\mathrm{EB}}(\mathrm{d})$ dependence for ferrihydrite in more detail after obtaining additional data on larger particles, which will by the object of the next study. 


\section{Conclusions}

Thus, we studied the effect of cooling in an external field with different values starting from the temperature exceeding the SP blocking temperature on the shift of magnetic hysteresis loops in the system of AF ferrihydrite nanoparticles with a size of $\sim(2.5-5 \mathrm{~nm})$. The shift of hysteresis loops was observed in all the investigated samples; however, in virtue of high fields of irreversibility of ZFC hysteresis loops, an important role in the observed shift of the $\mathrm{M}(\mathrm{H})$ dependence relative to the origin of coordinates is played by the minor hysteresis loop effect. This effect is significant at the external field cycling performed after field cooling. However, having compared the observed shift of the hysteresis loop with the extrapolated coercivity $\mathrm{H}_{\text {Cinf }}$ for a «limit» ZFC hysteresis loop, we may conclude that the exchange bias, which is not related to the minor hysteresis loop effect, exists in ferrihydrite nanoparticles. It is stably detected for the particles with an SP blocking temperature of $40 \mathrm{~K}$ and more. Note that the result obtained does not contradict the conclusions made in [26], where the exchange bias was not found in the ferritin and ferrihydrite samples with blocking temperatures of no more than $\sim 30 \mathrm{~K}$.

Based on the experimental data obtained, we proposed to determine the exchange bias $\mathrm{H}_{\mathrm{EB}}$ as a difference between $\mathrm{H}_{\mathrm{CL}}$ (coercivity of the «left» part of the hysteresis under the FC

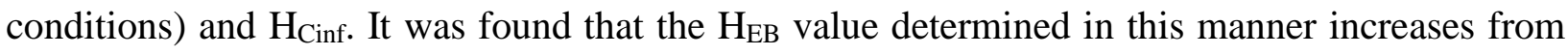
$\sim 0.3$ to $\sim 3 \mathrm{kOe}$ with an increase in the particle size from $\sim 3$ to $\sim 5 \mathrm{~nm}$ and, consequently, with an increase in $\mathrm{T}_{\mathrm{B}}$ from 40 to $90 \mathrm{~K}$. The latter is consistent with the data obtained for $\mathrm{CuO}$ and $\mathrm{NiO}$ nanoparticles $[7,8]$, which indicates the possible similarity of the exchange bias mechanisms.

\section{Acknowledgements}

The authors are grateful to M. Volochaev for measurements and O.A. Bayukov for Mössbauer study.

Table 1. Some parameters of the investigated samples of synthetic (s-fh) and bacterial (b-fh, bfh-a, and $\left.b-f h-a^{*}\right)$ ferrihydrite, including average particle size $\langle d\rangle$, blocking temperatures $T_{B}$ and $\left\langle\mathrm{T}_{\mathrm{B}}\right\rangle$, coercivity $\mathrm{H}_{\text {Cinf }}$ obtained by extrapolation of the $\mathrm{H}_{\mathrm{C}}\left(\mathrm{H}_{\max }\right)$ data at $\mathrm{T}=4.2 \mathrm{~K}$ by dependence (1), coercivity $\mathrm{H}_{\mathrm{CL}}$ under the FC conditions (the example of determination is shown in Fig. 2a), and exchange bias $\mathrm{H}_{\mathrm{EB}}$ determined from formula (3)

\begin{tabular}{|c|c|c|c|c|c|c|}
\hline sample & $<\mathrm{d}>, \mathrm{nm}$ & $\mathrm{T}_{\mathrm{B}}, \mathrm{K}$ & $\left\langle\mathrm{T}_{\mathrm{B}}\right\rangle, \mathrm{K}$ & $\mathrm{H}_{\text {Cinf }}, \mathrm{kOe}$ & $\mathrm{H}_{\mathrm{CL}}, \mathrm{kOe}$ & $\mathrm{H}_{\mathrm{EB}}, \mathrm{kOe}$ \\
\hline b-fh & 2.5 & 23.5 & 12.5 & $2.25 \pm 0.1$ & 2.34 & 0 \\
\hline s-fh & 3.0 & 40 & 12 & $4.5 \pm 0.15$ & 4.85 & 0.35 \\
\hline b-fh-a & 4.0 & 60 & 24.4 & $4.75 \pm 0.15$ & 6.0 & 1.25 \\
\hline b-fh-a* & 5.0 & 90 & 39 & $10.5 \pm 0.4$ & 13.8 & 3.3 \\
\hline
\end{tabular}




\section{References}

1. W.P. Meiklejohn, C.P. Bean, Phys. Rev. 102, 1413 (1956).

2. J. Nogués, I.K. Schuller, JMMM 192, 203 (1999).

3. J. Nogués, J. Sort, V. Langlais, V. Skumryev, S. Suriñach, J.S. Muñoz, M.D. Baró, Physics Reports 422, 65 (2005).

4. S. Giri, M. Patra and S. Majumdar, J. Phys.: Condens. Matter 23, 073201 (2011).

5. H. Bi, Sh. Li, Y. Zhang, Y. Du, JMMM 277, 363, (2004).

6. S.A. Makhlouf, F.T. Parker, F.E. Spada, and A.E. Berkowitz, J. Appl. Phys. 81 (8), 5561 (1997).

7. S.A. Makhlouf, H. Al-Attar, R.H. Kodama, Solid State Commun. 145, 1 (2008).

8. M.S. Seehra, A. Punnoose, Solid State Commun. 128, 299 (2003).

9. C. Diaz-Guerra, M. Vila, and J. Piqueras, Appl. Phys. Lett. 96193105 (2010).

10. A. Punnoose and M.S. Seehra, J. Appl. Phys. 91 (10), 7766 (2002).

11. A. Punnoose, H. Magnone, and M. S. Seehra, J. Bonevich, Phys. Rev. B 64, 174420 (2001).

12. J.F.K. Cooper, A. Ionescu, R.M. Langford, K.R.A. Ziebeck, C.H.W. Barnes, R. Gruar, C. Tighe, J.A. Darr, N.T.K. Thanh, and B. Ouladdiaf, J. Appl. Phys. 114083906 (2013).

13. A.E. Bianchi, S.J. Stewart, R.D. Zysler, and G. Punte, J. Appl. Phys. 112083904 (2012).

14. R.H. Kodama, A.E. Berkowitz, Phys. Rev. B 59, 6321 (1999).

15. S.A. Makhlouf, F.T. Parker, A.E. Berkowitz, Phys. Rev. B 55, R14717 (1997).

16. M.S. Seehra, V.S. Babu, A. Manivannan, J.W. Lynn, Phys. Rev. B 61, 3513 (2000).

17. S. Mørup, D.E. Madsen, C. Fradsen, C.R.H. Bahl, and M.F. Hansen, J. Phys.: Condens. Matter. 19, P. 213202 (2007).

18. Yu. L. Raikher and V. I. Stepanov, J. Exp. Theor. Phys. 107 (3), 435 (2008).

19. A. Punnoose, T. Phanthavady, M.S. Seehra, N. Shah, and G.P. Huffman, Phys. Rev. B 69, 054425 (2004).

20. T.S. Berquó, J.J. Erbs, A. Lindquist, R.L. Penn, and S.K. Banerjee, J. Phys.: Condens. Matter 21, 176005 (2009).

21. M.S. Seehra, V. Singh, X. Song, S. Bali, E.M. Eyring, Journal of Physics and Chemistry of Solids 711362 (2010)

22. H. Tüysüz, E.L. Salabas, Claudia Weidenthaler, and F. Schüth, J. Am. Chem. Soc., 130, 280 (2008).

23. D.A. Balaev, A.A. Dubrovskii, A.A. Krasikov, S.V. Stolyar, R.S. Iskhakov, V.P. Ladygina, and E.D. Khilazheva, JETP Lett. 98 (3), 139 (2013).

24. D.A. Balaev, A.A. Krasikov, A.A. Dubrovskii, S.V. Semenov, O.A. Bayukov, S.V. Stolyar, R.S. Iskhakov, V.P. Ladygina, and L.A. Ishchenko, J. Exp. Theor. Phys. 119 (3), 479 (2014).

25. D.A. Balaev, A.A. Krasikov, A.A. Dubrovskiy, S.V. Semenov, S.I. Popkov, S.V. Stolyar, R.S. Iskhakov, V.P. Ladygina, and R.N. Yaroslavtsev, Phys. Solid State 58 (2) 287 (2016).

26. N.J.O. Silva, V.S. Amaral, A. Urtizberea, R. Bustamante, A. Millán, F. Palacio, E. Kampert and U. Zeitler, S. de Brion, O. Iglesias and A. Labarta, Phys. Rev. B 84, 104427 (2011).

27. N.J.O. Silva, V.S. Amaral, L.D. Carlos, and V. de Zea Bermudez, J. Appl. Phys. 93 (10), 6978 (2003).

28. N.J.O. Silva, V.S. Amaral, L.D. Carlos, B. Rodríguez-González, M. Liz-Marzán, A. Millan, F. Palacio, and V. de Zea Bermudez, J. Appl. Phys. 100, 054301 (2006).

29. S.V. Stolyar, O.A. Bayukov, Yu.L. Gurevich, V.P. Ladygina, R. S. Iskhakov, and P. P. Pustoshilov, Inorg. Mater. 41 (6), 638 (2007).

30. Yu.L. Raikher, V.I. Stepanov, S.V. Stolyar, V.P. Ladygina, D.A. Balaev, L.A. Ishchenko, and M. Balasoiu, Phys. Solid State 52 (2), 298 (2010). 
31. D.A. Balaev, A.A. Krasikov, A.A. Dubrovskii, O.A. Bayukov, S.V. Stolyar, R.S. Iskhakov, V.P. Ladygina, and R.N. Yaroslavtsev, Tech. Phys. Lett. 41 (7), 705 (2015).

32. D.A. Balaev, A.A. Krasikov, A.A. Dubrovskiy, S.I. Popkov, S.V. Stolyar, O.A. Bayukov, R.S. Iskhakov, V.P. Ladygina, R.N. Yaroslavtsev, JMMM 410, 71 (2016).

33. F.M. Michel, L. Ehm, S.M. Antao, P.L. Lee, P.J. Chupas, G. Liu, D.R. Strongin, M.A.A. Schoonen, B.L. Phillips, J.B. Parise, Science 316, 1726 (2007).

34. D.A. Balaev, A.A. Krasikov, S.V. Stolyar, R.S. Iskhakov, et. al, Phys. Solid State, in press (2016)

35. A.D. Balaev, Yu.V. Boyarshinov, M.M. Karpenko, and B.P. Khrustalev, Prib. Tekh. Eksp., 3, 167 (1985).

36. D. Tobia, E. Winkler, R.D. Zysler, M. Granada, H.E. Troiani, and D. Fiorani, Journ. Appl. Phys. 106, 103920 (2009).

37. C. Binek, Phys. Rev. B 70, 014421 (2004).

38. Zh. Tian, Ch. Zhu, Y. Liu, J. Shi, Zh. Ouyang, Zh. Xia, G. Du, and S. Yuan, J. Appl. Phys. 115, 083902 (2014).

39. R.P. Guertin, N. Harrison, Z.X. Zhou, S. McCall, F. Drymiotis, J. Magn. Magn. Mater. 308, 97 (2007). 50Tesla

40. N.J.O. Silva, A. Millán, and F. Palacio, E. Kampert and U. Zeitler, H. Rakoto, V.S. Amaral, Phys. Rev. B 79, 104405 (2009).

41. L. Néel, C.R. Acad. Sci. Paris 252, 4075 (1961).

42. N.J.O. Silva, V.S. Amaral, and L.D. Carlos, Phys. Rev. B 71, 184408 (2005).

43. J.G.E. Harris, J.E. Grimaldi, D.D. Awschalom, A. Chiolero, and D. Loss, Phys. Rev. B 60, 3453 (1999).

\section{Figure captions.}

Fig. 1. (Color online) Temperature dependences of the magnetic moment for the investigated samples in an external field of $\mathrm{H}=1 \mathrm{kOe}$ in the $\mathrm{FC}$ and $\mathrm{ZFC}$ modes.

Fig. 2. (Color online) ZFC hysteresis loops at $\mathrm{T}=4.2 \mathrm{~K}$ together with $\mathrm{FC}$ hysteresis loops for the investigated samples. Insets in $2 \mathrm{a}, 2 \mathrm{~b}$, and $2 \mathrm{c}$ show the portions of the $\mathrm{M}(\mathrm{H})$ dependences near the origin of coordinates. Inset in $2 \mathrm{a}$ explains determination of parameters $\mathrm{H}_{\mathrm{CL}}, \mathrm{H}_{\mathrm{CR}}$, and $\mathrm{M}_{\mathrm{R}}$ of the shifted hysteresis loops.

Fig. 3. (Color online) Evolution of (a, c, and d) the FC hysteresis loops at different $\mathrm{H}_{\text {cool }}$ values and subsequent external field cycling within $\pm \mathrm{H}_{\text {cool }}$ and (b) minor hysteresis loops after applying a field of $\mathrm{H}=+75 \mathrm{kOe}$ and subsequent field cycling within $\pm 15 \mathrm{kOe}$. (e) behavior of parameters $\mathrm{H}_{\mathrm{CL}}$ and $\mathrm{H}_{\mathrm{CR}}$ from $3 \mathrm{a}-3 \mathrm{~d}$. $\mathrm{T}=4.2 \mathrm{~K}$.

Fig. 4. (Color online) Dependences of $H_{C}$ (on the top) and $M_{R}$ (in the bottom) on $\left|H_{\max }\right|$ for samples s-fh, b-fh-a, and b-fh obtained from the ZFC hysteresis loops at $4.2 \mathrm{~K}$. Solid lines indicate the best fittings by expressions (1) and (2) for obtaining the $\mathrm{H}_{\mathrm{Cinf}}$ and $\mathrm{M}_{\mathrm{Rinf}}$ values shown by the horizontal dashed lines. Data on $\mathrm{H}_{\mathrm{CL}}, \mathrm{H}_{\mathrm{CR}}$, and $\mathrm{M}_{\mathrm{R}}$ for the $\mathrm{FC}$ hysteresis loops are built at the abscissas corresponding to $\mathrm{H}_{\text {cool. }}$. For the minor hysteresis loop regime, (ML(75kOe), see Section 2.2), the abscissa is taken to be $15 \mathrm{kOe}$. 
Fig. 5. (Color online). ZFC hysteresis loops at $\mathrm{T}=4.2 \mathrm{~K}$ together with $\mathrm{FC}$ hysteresis loops at $\mathrm{H}_{\mathrm{cool}}=30 \mathrm{kOe}$ for sample $\mathrm{b}-\mathrm{fh}-\mathrm{a}^{*}$. Pairs of points at $\mathrm{M}=0$ and $\mathrm{H}=0$ correspond to coercivity $\pm \mathrm{H}_{\text {Cinf }}$ and residual moment $\pm \mathrm{M}_{\text {Rinf }}$ of the «limit» hysteresis loop. The left inset shows these data in the enlarged scale. The right inset shows the example of obtaining the $\mathrm{H}_{\text {Cinf }}$ value by fitting the $\mathrm{H}_{\mathrm{C}}\left(\mathrm{H}_{\max }\right)$ dependence using expression (1). The horizontal straight corresponds to the $\mathrm{H}_{\mathrm{Cinf}}$ value and a set of points with an abscissa of $\mathrm{H}=30 \mathrm{kOe}$ corresponds to the evolution of the $\mathrm{H}_{\mathrm{CL}}$ values at the field cycling within $\pm 30 \mathrm{kOe}$ after cooling at $\mathrm{H}_{\mathrm{cool}}=30 \mathrm{kOe}$. 


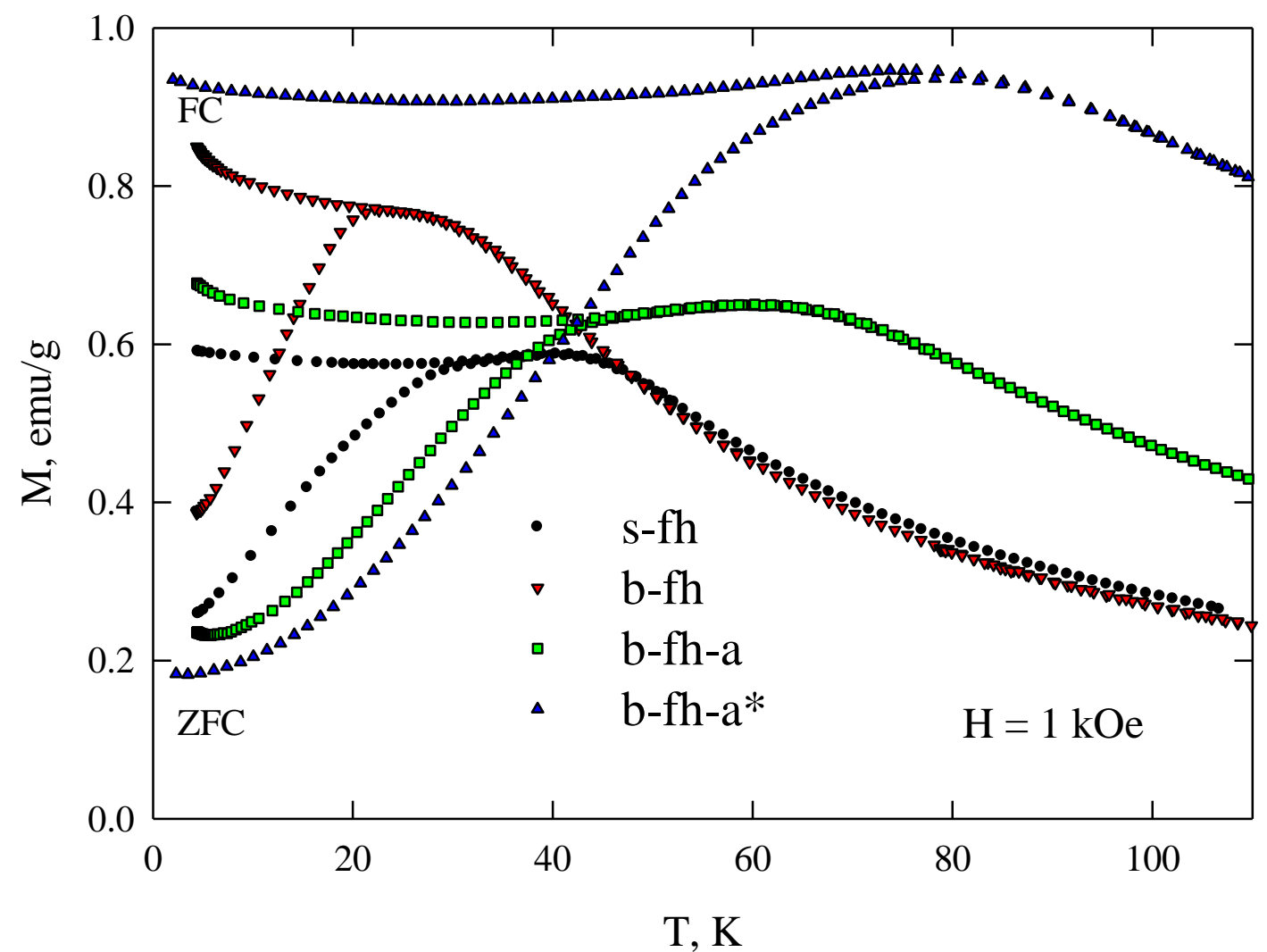

Fig. 1. (Color online) Temperature dependences of the magnetic moment for the investigated samples in an external field of $\mathrm{H}=1 \mathrm{kOe}$ in the $\mathrm{FC}$ and ZFC modes. 


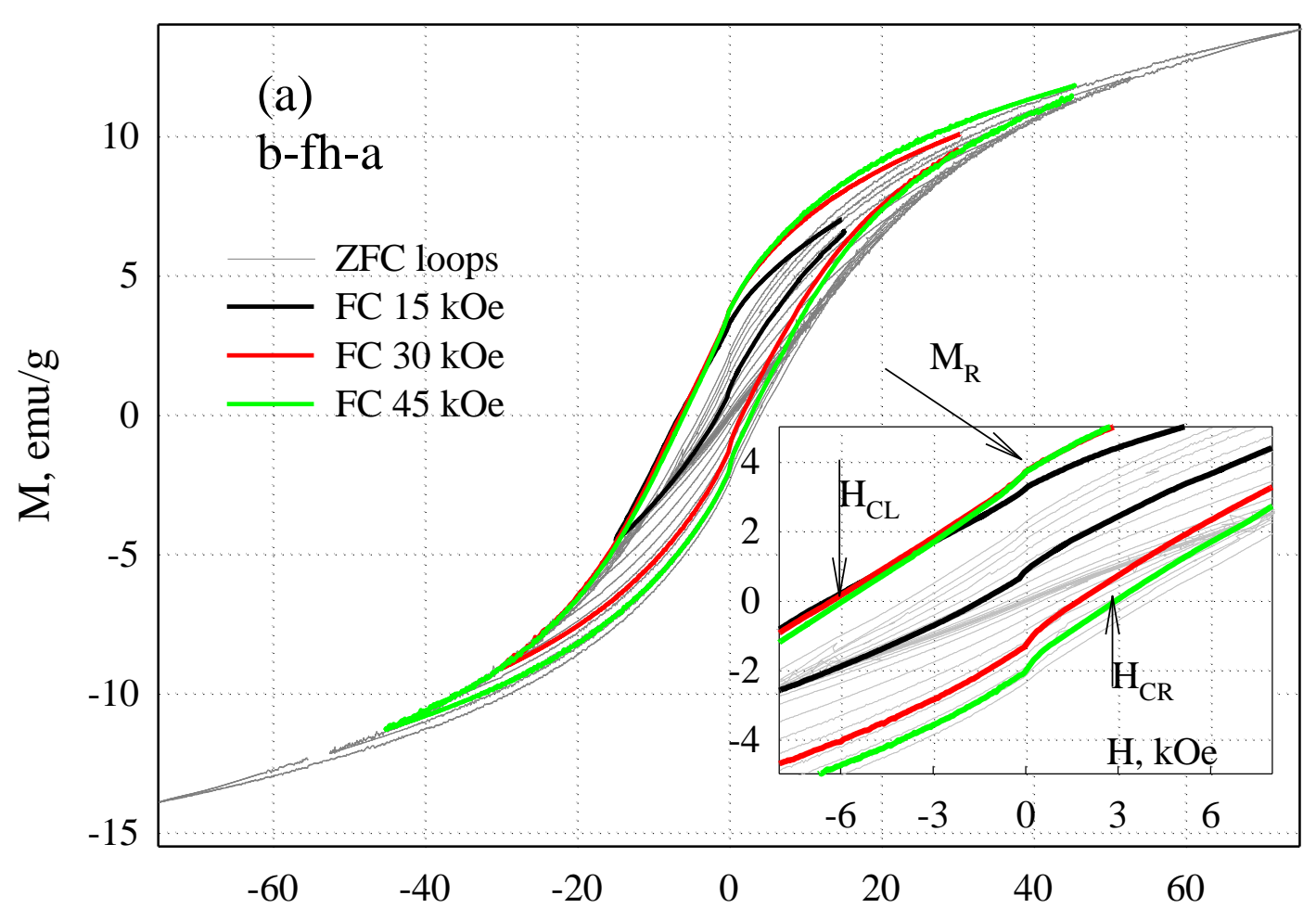

$\mathrm{H}, \mathrm{kOe}$

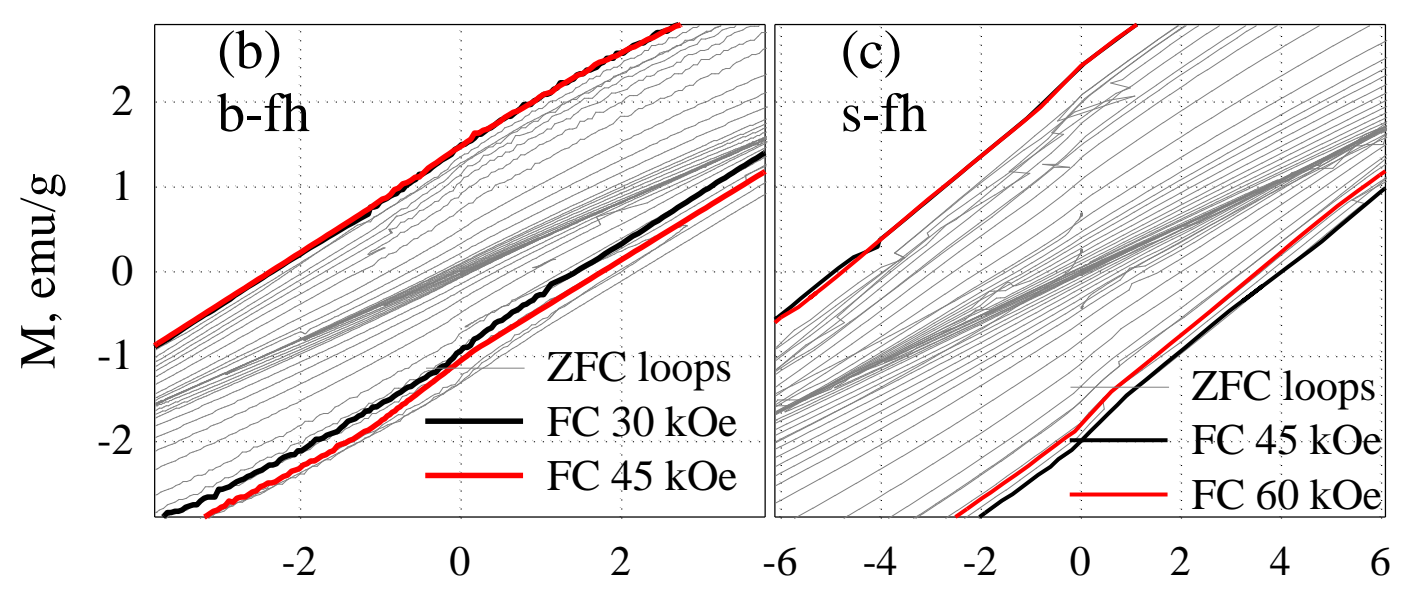

$\mathrm{H}, \mathrm{kOe}$

$\mathrm{H}, \mathrm{kOe}$

Fig. 2. (Color online) ZFC hysteresis loops at $\mathrm{T}=4.2 \mathrm{~K}$ together with $\mathrm{FC}$ hysteresis loops for the investigated samples. Insets in $2 a, 2 b$, and $2 c$ show the portions of the $M(H)$ dependences near the origin of coordinates. Inset in $2 \mathrm{a}$ explains determination of parameters $\mathrm{H}_{\mathrm{CL}}, \mathrm{H}_{\mathrm{CR}}$, and $\mathrm{M}_{\mathrm{R}}$ of the shifted hysteresis loops. 


\section{b-fh-a}
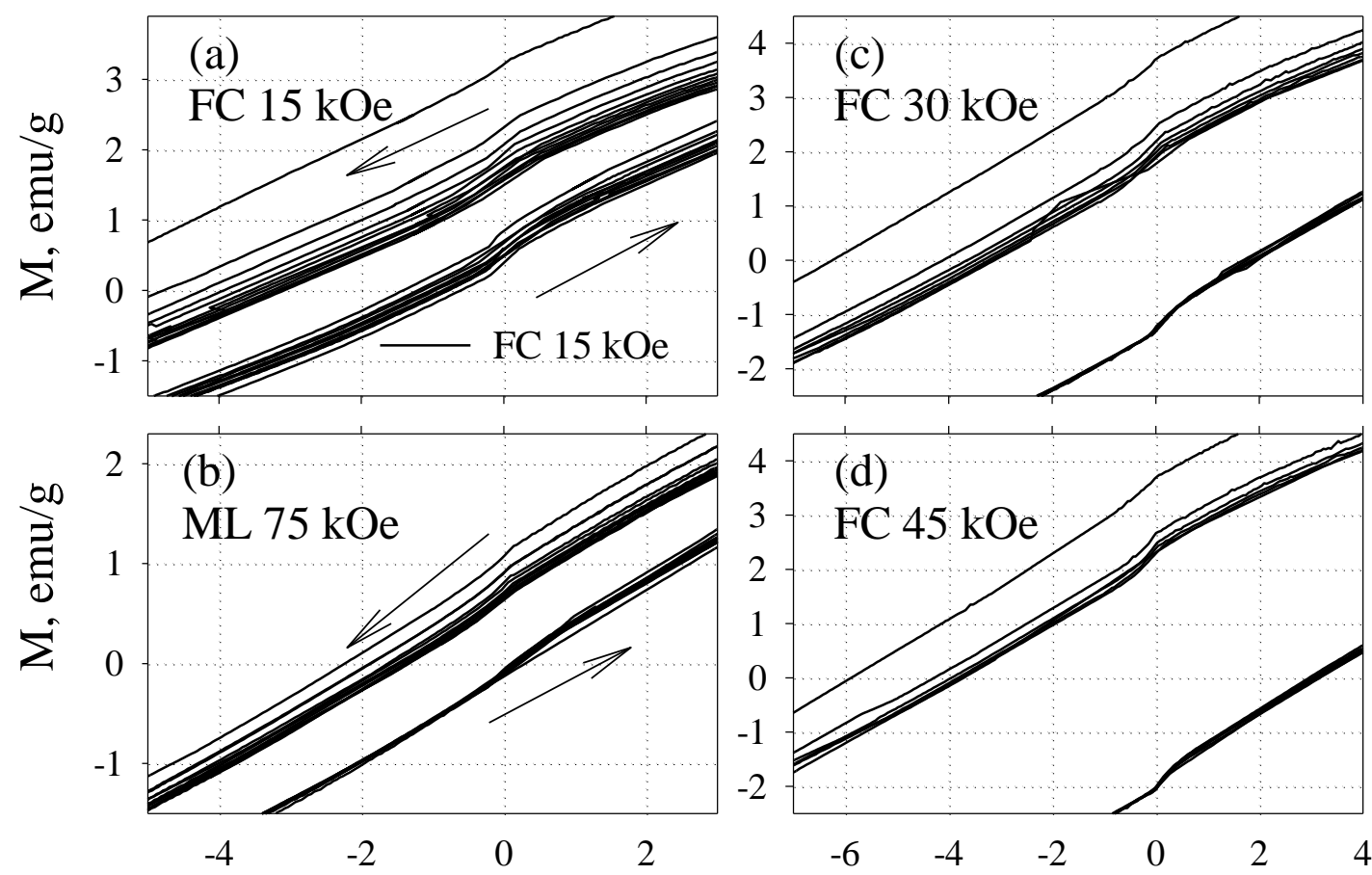

$\mathrm{H}, \mathrm{kOe}$

$\mathrm{H}, \mathrm{kOe}$

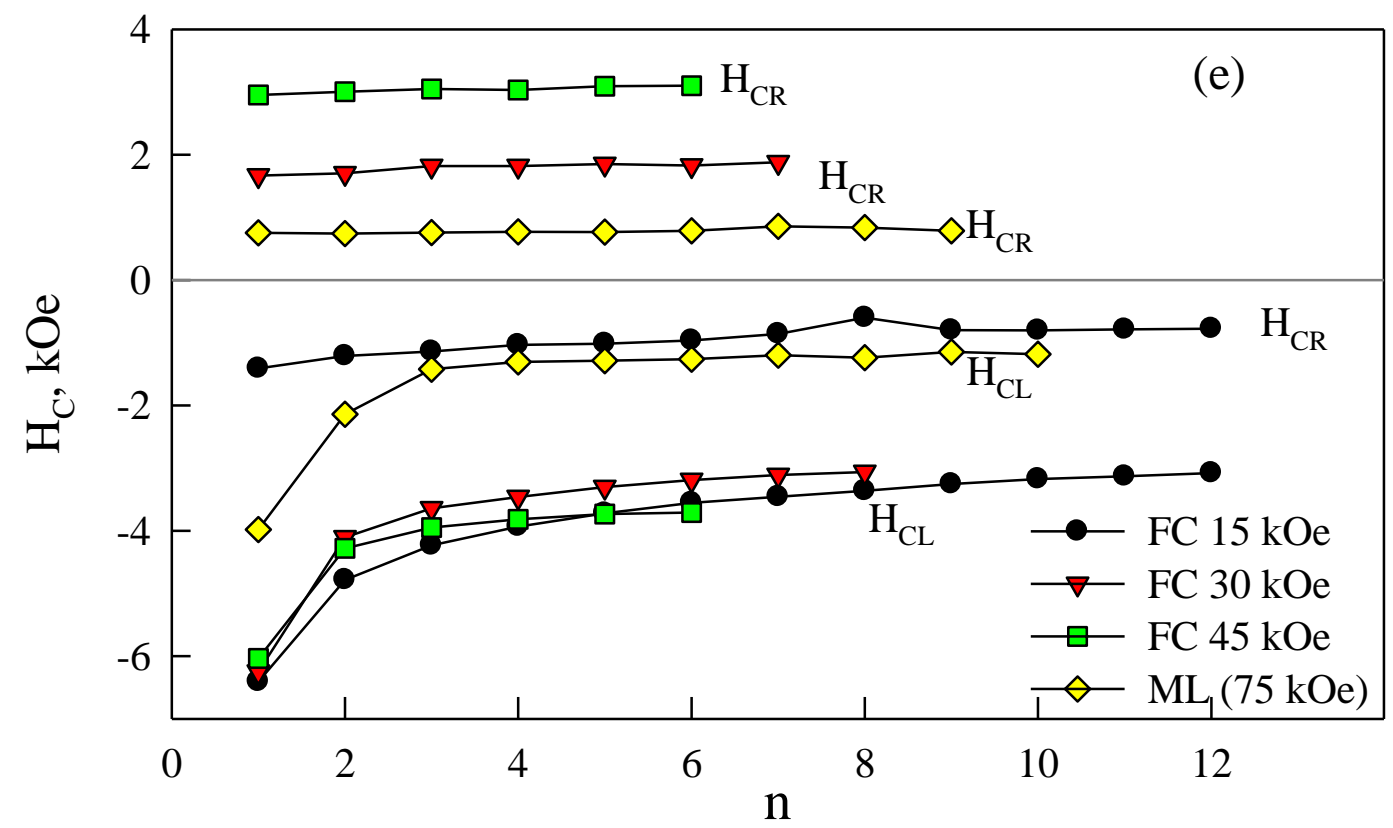

Fig. 3. (Color online) Evolution of (a, c, and d) the FC hysteresis loops at different $\mathrm{H}_{\text {cool }}$ values and subsequent external field cycling within $\pm \mathrm{H}_{\text {cool }}$ and (b) minor hysteresis loops after applying a field of $\mathrm{H}=+75 \mathrm{kOe}$ and subsequent field cycling within $\pm 15 \mathrm{kOe}$. (e) behavior of parameters $\mathrm{H}_{\mathrm{CL}}$ and $\mathrm{H}_{\mathrm{CR}}$ from $3 \mathrm{a}-3 \mathrm{~d}$. $\mathrm{T}=4.2 \mathrm{~K}$. 


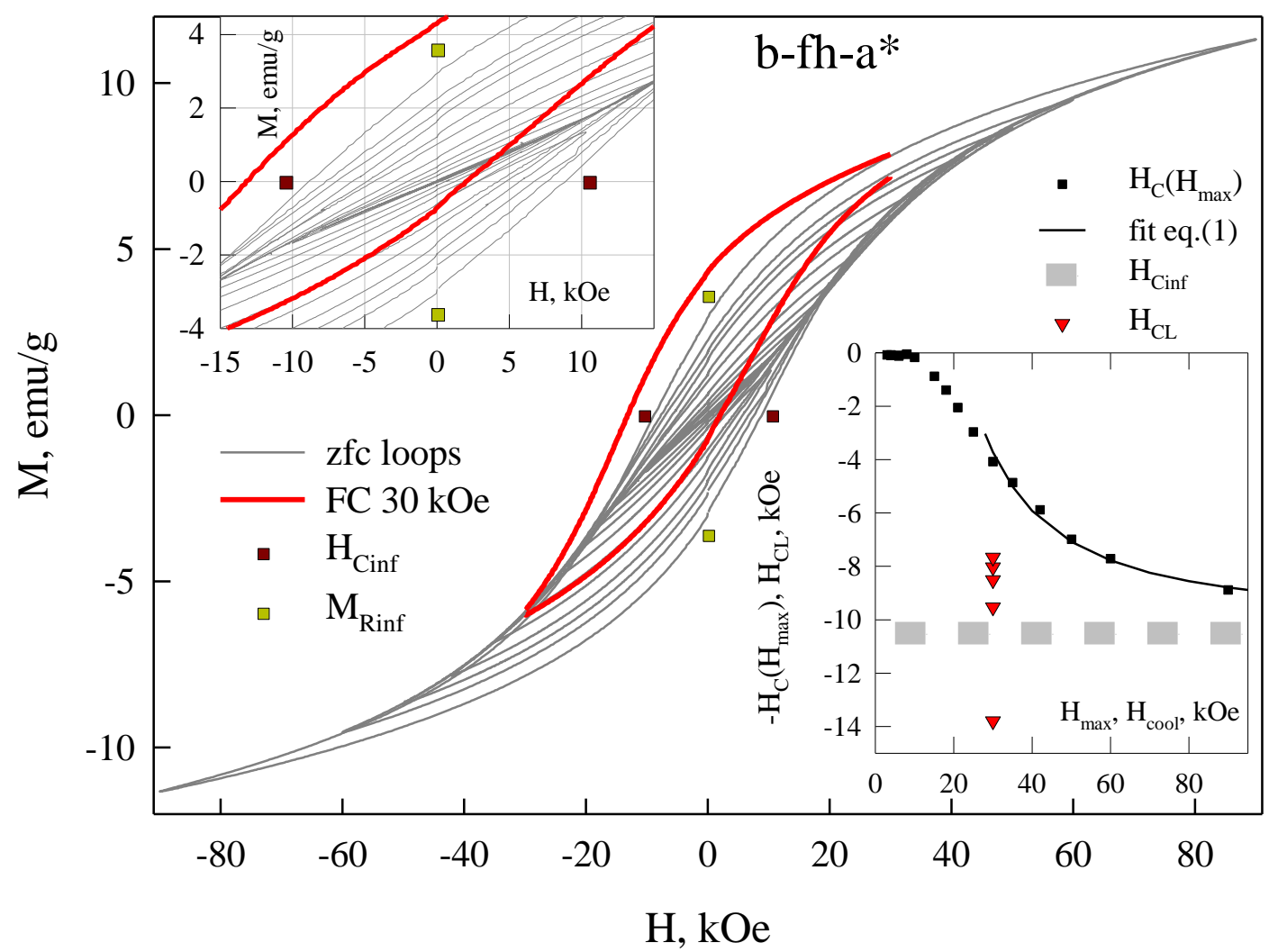

Fig. 5. (Color online). ZFC hysteresis loops at $\mathrm{T}=4.2 \mathrm{~K}$ together with $\mathrm{FC}$ hysteresis loops at $\mathrm{H}_{\mathrm{cool}}=30 \mathrm{kOe}$ for sample $\mathrm{b}-\mathrm{fh}-\mathrm{a}^{*}$. Pairs of points at $\mathrm{M}=0$ and $\mathrm{H}=0$ correspond to coercivity $\pm \mathrm{H}_{\text {Cinf }}$ and residual moment $\pm \mathrm{M}_{\text {Rinf }}$ of the «limit» hysteresis loop. The left inset shows these data in the enlarged scale. The right inset shows the example of obtaining the $\mathrm{H}_{\mathrm{Cinf}}$ value by fitting the $\mathrm{H}_{\mathrm{C}}\left(\mathrm{H}_{\max }\right)$ dependence using expression (1). The horizontal straight corresponds to the $\mathrm{H}_{\text {Cinf }}$ value and a set of points with an abscissa of $\mathrm{H}=30 \mathrm{kOe}$ corresponds to the evolution of the $\mathrm{H}_{\mathrm{CL}}$ values at the field cycling within $\pm 30 \mathrm{kOe}$ after cooling at $\mathrm{H}_{\mathrm{cool}}=30 \mathrm{kOe}$.

Fig. 4. (Color online) Dependences of $H_{C}$ (on the top) and $M_{R}$ (in the bottom) on $\left|H_{\max }\right|$ for samples s-fh, b-fh-a, and b-fh obtained from the ZFC hysteresis loops at $4.2 \mathrm{~K}$. Solid lines indicate the best fittings by expressions (1) and (2) for obtaining the $\mathrm{H}_{\text {Cinf }}$ and $\mathrm{M}_{\text {Rinf }}$ values shown by the horizontal dashed lines. Data on $\mathrm{H}_{\mathrm{CL}}, \mathrm{H}_{\mathrm{CR}}$, and $\mathrm{M}_{\mathrm{R}}$ for the $\mathrm{FC}$ hysteresis loops are built at the abscissas corresponding to $\mathrm{H}_{\text {cool }}$. For the minor hysteresis loop regime, (ML(75kOe), see Section 2.2), the abscissa is taken to be $15 \mathrm{kOe}$. 

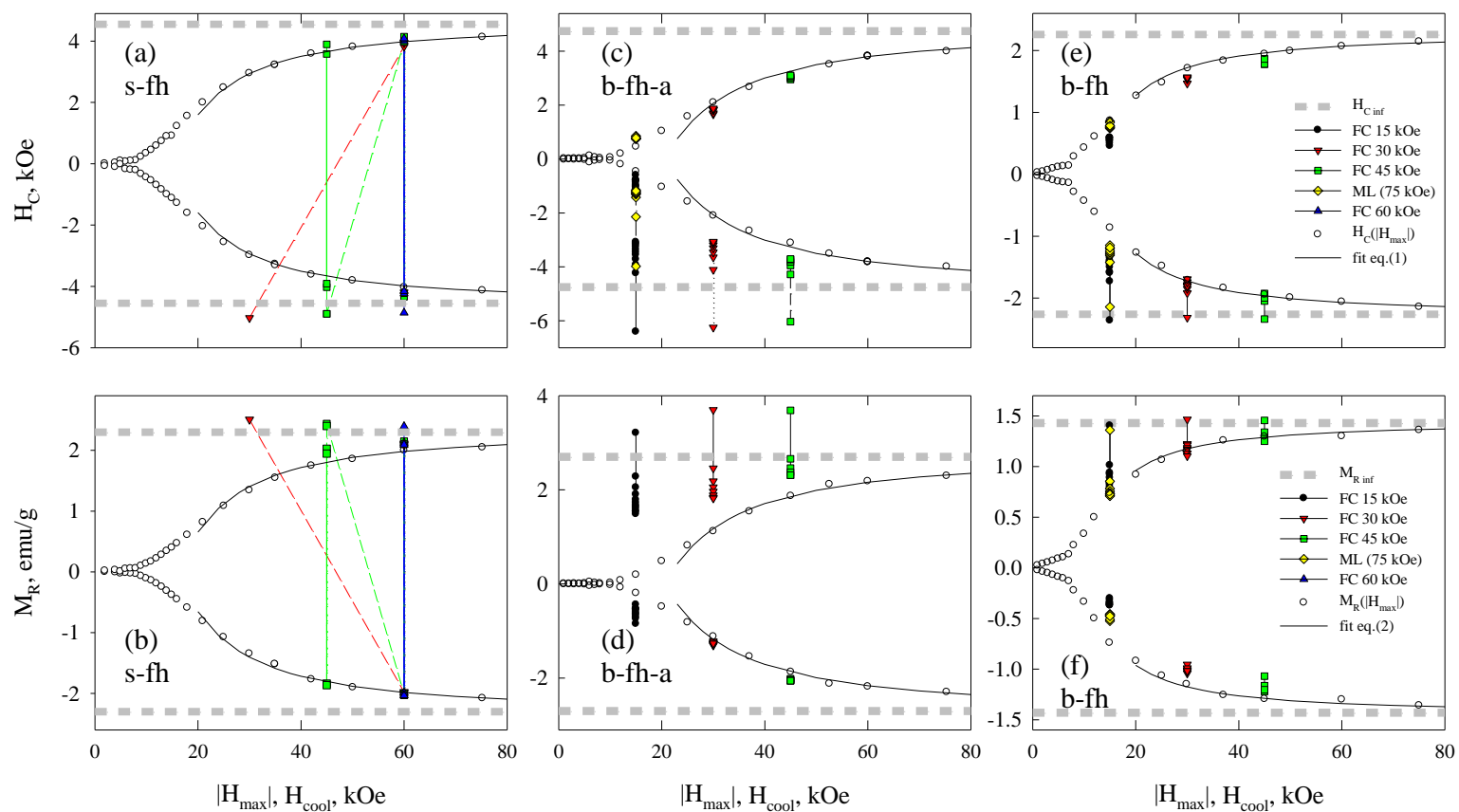

Fig. 4. 